\title{
A Comparison of Common Methods for Optimal Well Placement
}

\author{
Jeremy J. Minton \\ Supervised by: Associate Professor Rosalind Archer \\ Department of Engineering Science, Faculty of Engineering, University of Auckland, New Zealand
}

\begin{abstract}
The well placement problem is challenging due to the non-linear, discrete and often multi-modal objective function. This is complicated by computationally expensive function evaluations from reservoir simulation, typically producing no gradient information. Heuristics for automated optimisation have been proposed over the past 20 years with minimal comparison or benchmarking of performance. This paper presents a comparison of common methods, including genetic algorithms, simulated annealing, particle swarm optimisation and variants of the hill climbing algorithms. These algorithms were tested in the context of locating a single production or injection well in two reservoir cases. For this class of problem, the genetic algorithm produced good results after 100 evaluations. The particle swarm method performed only slightly worse but was able to improve considerably with the use of 'educated guesses' seeding it's initialisation. For this reason the particle swarm optimiser is arguably the better method for industrial implementation as some idea of optimality would exist through intuition and experience. A recommendation for further development is the investigation of objective function approximations for initialisation seeding as well as sequentially combining algorithms, such as particle swarm and simulated annealing, to find a combination of explorative then exploitative searching. The validity of these results are limited, however, due to the small sample size and to single well problems. In fact, some findings were contradictory to previously published guidelines which illustrates the need for this type of comparison: understanding an algorithm's performance under given conditions is necessary in leveraging its benefits, particularly with such demanding problems such as well placement.
\end{abstract}

Abstract

Keywords:

well placement, algorithm comparison, heuristics, particle swarm optimisation, genetic algorithm, simulated annealing, hill climbing

Email:

jmin040@aucklanduni.ac.nz (Jeremy Minton) 


\section{Introduction}

Selecting optimal well sites is a valuable problem to solve; maximising oil recovery increases oil reserves and minimising costs improves profitability. Unfortunately, the non-linear and multi-modal response of well locations and the discrete nature of most decision variables through the discretisation of the reservoir, make this a challenging optimisation problem for deterministic searches. Furthermore, the function evaluation involves computational simulation of the fluid flow within the reservoir; typically called reservoir simulation. The high computational cost of performing these reservoir simulations increases the demand for an algorithm to find optimality with the fewest function evaluations.

Consequently, since 1995, research has been focused on developing heuristics to rapidly solve reservoir simulation models for near optimality (Aanonsen et al., 1995; Beckner and Song, 1995). This topic has been gaining popularity as computer processing speed has improved the feasibility of these investigations.

Unfortunately, the problem is dependent on properties of the particular reservoir: heterogeneity will increase the noise in the objective function and produce larger and more local optima; the number of wells to be placed can be introduced as a control variable which creates a stepped objective function; similarly, a variable number of lateral wells or completion intervals can be optimised. Each subsequent layer of complexity makes the problem more difficult to solve and alters the efficiency of any algorithm configuration used.

The high computational cost of reservoir simulation implies that the complexity of the algorithm is negligible compared to function evaluations. Consequently, many methods have been proposed.

\subsection{Published Algorithms}

Interpolation methods were one of the first methods applied to the well placement problem, having been adapted from the history matching problem. Examples of the surface approximation techniques used include Kriging, least squares and regression surfaces (Pan and Horne, 1998; Aanonsen et al., 1995).

Simulated annealing, is a meta-heuristic that is based on the annealing process in metallurgy. It was, also, one of the first published methods, applied to the well placement problem by Beckner and Song (1995).

Evolutionary algorithms use a process of survival of the fittest: better solutions have a higher probability of 'mating' and hence their characteristics are more likely to persist into later iterations. Evolutionary strategies are currently the most popular methods with 2,260 results of 8,840 found in the OnePetro database on 'well placement optimisation' also containing the term "genetic" or "evolution". Some relevant papers for the interested reader include Montes et al. (2001); Tupac et al. (2007); Morales et al. (2011); Ding (2008).

Particle swarm optimisation (PSO) was originally designed by Kennedy and Eberhart (1995) as a continuous non-linear function optimiser and has had a range of developments and applications as summarised in Poli et al. (2007). It has been applied to the well placement problem in three publications; Onwunalu and Durlofsky (2009a), Onwunalu and Durlofsky (2009b) and Ciaurri et al. (2011).

A number of publications have been made regarding variations of the descent algorithms. These include derivative free hill climbing such as stochastic directions, pattern searches, direct searches as well as derivative methods like finite differences or adjoint gradient estimation methods (Bangerth et al., 2006; Onwunalu and Durlofsky, 2009b; Zandvliet et al., 2008; Sarma and Chen, 2008).

An assortment of other less common algorithms have also been posed. Wang et al. (2007) presents a novel approach in which flow rates of wells at every grid point are optimised and wells are eliminated as their flow rate drops to zero. A method to optimise lateral wells based on quality maps, a guide to regions of high production potential, is presented in Nakajima and Schiozer (2003). Lo et al. (1995) developed a linear forecasting model to harness linear programming techniques. Finally, Ciaurri et al. (2011) identifies asynchronous parallel pattern search, implicit filtering, dividing rectangles and some statistical emulation methods.

Additionally, many combination and hybrid methods have also been examined which combine different algorithms to exploit the high performing behaviours of each. Bittencourt and Horne (1997); Zangl et al. (2006); Badru and Kabir (2003); Guyaguler et al. (2002) are a few examples for the interested reader.

This is by no means an exhaustive list.

\subsection{Algorithm Comparison}

Despite the great disparity in the number of function evaluations required, ranging from tens to thousands in the previously cited papers, (Bangerth et al., 2006) is one of the few studies that compares algorithms: simultaneous perturbation stochastic approximation, finite difference gradient, very fast simulated annealing, Nelder-Mead simplex and a genetic algorithm in this case. Performing this style of comparison with a sufficiently comprehensive selection of algorithms on case studies representative of industrial problems would fill an apparent gap in published literature. Through better understanding the behaviour and performance, the improvement and even development of new, novel algorithms could be directed.

To this end, this study investigates the application of four optimisation algorithms in two well placement optimisation problems. Due to computational restraints the problems were restricted to locating a single vertical 
well, defined by two surface indices and the bottommost perforation depth. This allowed an exhaustive search to be performed so multiple trials could be completed for statistical validity. The algorithms tested were the descent method, simulated annealing, genetic algorithm and particle swarm optimisation.

\section{Methodology}

The algorithms compared in this study were tested optimising the location and depth of a single well in one synthetic and one realistic reservoir model. The problem formulation and test cases follow in this section. The algorithms, described in the next section, were implemented as constrained optimisation algorithms and each parameter set was used to optimise each well placement problem 200 times. These repetitions are to account for the algorithm's stochastic behaviour.

\subsubsection{Parameter Selection}

The performance of an algorithm in a given context will depend on the balance of exploitative and explorative behaviours. Identifying appropriate control parameters achieves this correct balance, however, this is not a trivial problem and they are rarely transferable between functions. Unfortunately, computational limits prevented an exhaustive search or optimisation of these parameters hence those identified as generally successful in literature were used. Some variations were also tested for assessing performance sensitivity. These are detailed with appropriate citations in Section 3.

\subsubsection{Seeded Initialisation}

It was identified in Zandvliet et al. (2008) that hill climbing has the advantage that it will improve at every iteration until the local optimum is found, whereas meta-heuristics may undergo numerous iterations without making any improvement. This suggests an algorithm's ability to utilise information from a previous iteration varies and hence it's ability to utilise information from intelligently selected initialisation points. The reservoir simulation process is not a black box; quickly calculable approximations and experientially based guesses contain large amounts of information about the system. Although not acceptable for optimisation, such approximations have merit by eliminating the obviously poor regions of the reservoir from the search space. Hence, effective use of 'a priori' knowledge would make the process attractive in the industrial environment; such an algorithm could be readily adopted into commercial use as a final tuning step in the conventional planning process.

This is tested in a second round of experiments by substituting otherwise randomly generated initial solutions with initial 'guesses' of the solution. The maximum of some function, based on some quickly attainable estimate of the objective function, is used, where one is randomly chosen when multiple maxima exist; these estimated solutions are referred to as optimal solution approximations or just approximations in this paper.

Once all approximations have been used to generate points, random selection continues. This means for a population algorithm, the first number of individuals are generated from approximations and the remaining are random and for single-point methods the first number of restarts are guided by approximations before restarting randomly.

These approximations, in priority for use, are:

1. Well performance index (WPI) The well performance index from a simulation of a very low production rate well at every cell for a single time-step.

2. Permeability The input permeability for each cell of the reservoir.

3. Porosity The input porosity for each cell of the reservoir.

4. Cell Thickness The vertical dimension of each cell in the reservoir.

5. Cell Depth The depth of the top of each cell in the reservoir.

6. Net to gross The input net to gross (refer to glossary) of each cell in the reservoir.

Each estimated function surface, from which the optimal solution approximations was selected, is compared to the objective function surfaces in Appendix B.

\subsection{Problem Formulation}

The optimisation problem used in this paper is:

\section{Decision Variables}

Grid block co-ordinates of the well head.

Layer index of the lower perforation limit (only considered for the three dimensional case). 
Objective Functions Two objective functions were considered independently.

Maximise cumulative oil production (COP) of the field or, maximise net present value (NPV) of the field.

\section{Constraints}

Boundary constraints - wells must be located within the reservoir simulation model.

\subsubsection{Objective Function Evaluation}

NPV is calculated using the formulation provided in Onwunalu and Durlofsky (2009b), reproduced here for convenience.

$$
\mathrm{NPV}=\sum_{t=1}^{T} \frac{C F_{t}}{(1+r)^{t}}-C^{\mathrm{capex}}
$$

where $r$ is the rate of return, $T$ is the set of time intervals, $C F_{t}$ is cash flow in the time period $t$ and capital expenditure, $C^{\text {capex }}$, is the cost of any development work and is defined as,

$$
C^{\text {capex }}=\sum_{w=1}^{N_{\text {well }}} C^{\text {surface }}+C^{\text {drill }} L^{\text {shaft }},
$$
feet.

where $N_{\text {well }}$ is the number of wells to be drilled, $C$ is the cost of the superscript and L is the well depth in

The cash flow term is revenue minus expenses,

$$
C F_{t}=p^{o} Q_{t}^{o}+p^{g} Q_{t}^{g}-C^{w p} Q_{t}^{w p}-C^{w i} Q_{t}^{w i},
$$

where $p$ is the price of oil or gas, $C$ is the cost of water production or water injection and $Q$ denotes the production or injection rates of each fluid during the denoted time interval.

The values used in these calculations are included in Table 1. For simplicity many financial considerations

Table 1: Values Used in the NPV Calculation

\begin{tabular}{c|c} 
Term & Value \\
\hline$r$ & $10 \%$ \\
$C^{\text {surface }}$ & $\$ 5,000,000$ per Well \\
$C^{\text {drill }}$ & $\$ 0$ per foot \\
$p^{o}$ & $\$ 22$ per stock tank barrel \\
$p^{g}$ & $\$ 1.5$ per thousand cubic feet \\
$C^{w p}$ & $\$ 1$ per stock tank barrel \\
$C^{w i}$ & $\$ 1$ per stock tank barrel
\end{tabular}

are omitted including the cost of drilling, cost forecasting, location dependent costs or more complex capital expenditure models. A more sophisticated class of model may improve accuracy but should not overly influence the response surface.

\subsection{Test Cases}

Using the 'ECLIPSE' reservoir simulator (ECLIPSE, 2009), an exhaustive search of the location and depth of one well over the reservoir models considered was performed, holding all other properties constant including cell layout, cell parameters and existing well configurations. This produced a response surface that shortened function evaluations to a single look-up operation. The response surfaces are presented in Appendix A.

\subsubsection{Synthetic Reservoir}

The synthetic reservoir used was 'SPE9'. After completion of a large study by the Society for Petroleum Engineers, this model was made available with the 'ECLIPSE' simulator. It is synthesised to be representative of a north sea formation, with uniform cell thickness and porosity by layer ranging from 8 to 100 feet and 0.08 to 0.157 respectively. Each cell's permeability is horizontally isotropic with the vertical permeability roughly one hundredth of this value; values range from less than 1 milli-Darcy to several Darcy throughout the reservoir. There is a water 


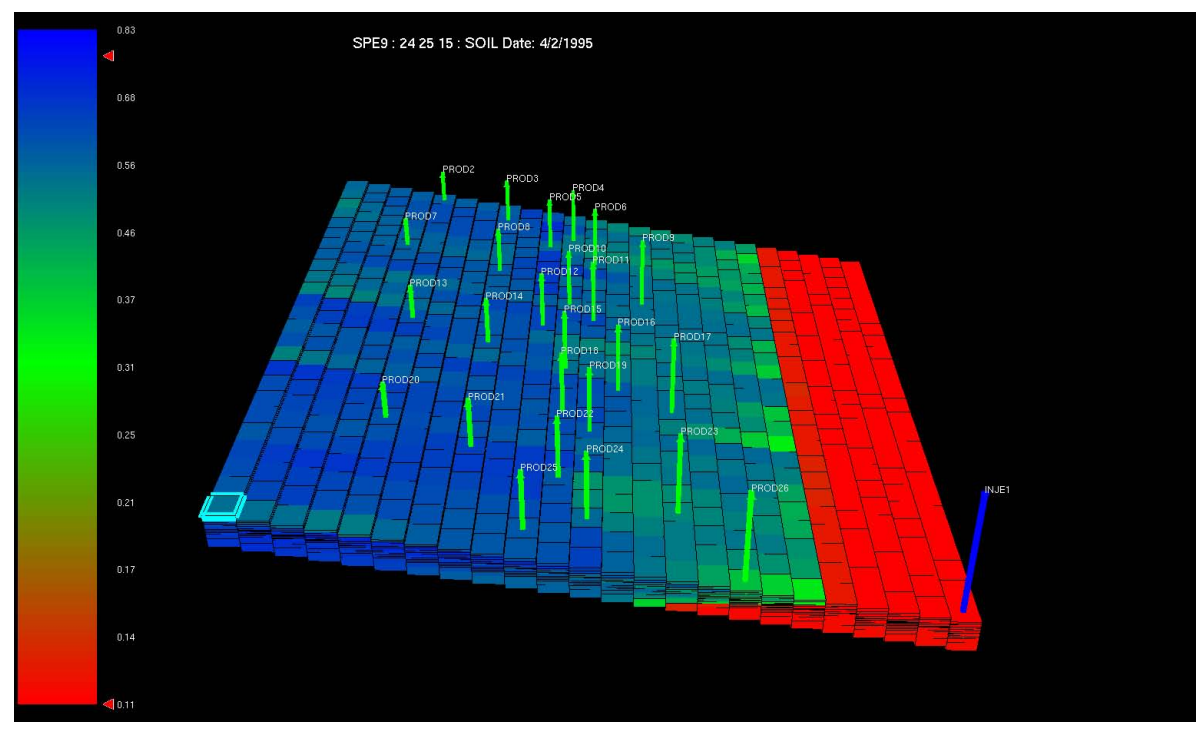

Figure 1: Three dimensional view of the synthetic reservoir showing the oil saturation; the blue and red colours indicating high and low saturation respectively.

leg, the low oil saturation at the bottom edge of the reservoir in Figure 1, but no gas cap. The model contains $24 \times 25 \times 15$ cells.

The model was used to locate one new production well amongst 26 existing producers and one water injection well. The perforation interval began from the second layer, limiting the three-dimensional optimisation to 14 layers. For the two dimensional trials the completion depth was taken as the tenth layer. Oil production was found to correlate with the oil saturation at the location of the new producer.

\subsubsection{Real-World Reservoir}

The realistic reservoir used contains $54 \times 55 \times 5$ cells. It is a black-oil model with uniform porosity of 0.26 in the first four layers and 0.15 in the bottom; uniform permeability of $20 \mathrm{mD}$ except a zone at $70 \mathrm{mD}$ near well 'N1' and another of $2 \mathrm{mD}$ about well ' $\mathrm{N} 8$ '. The structure of the reservoir, initial oil saturation and location of six existing production wells, is displayed in Figure 2.

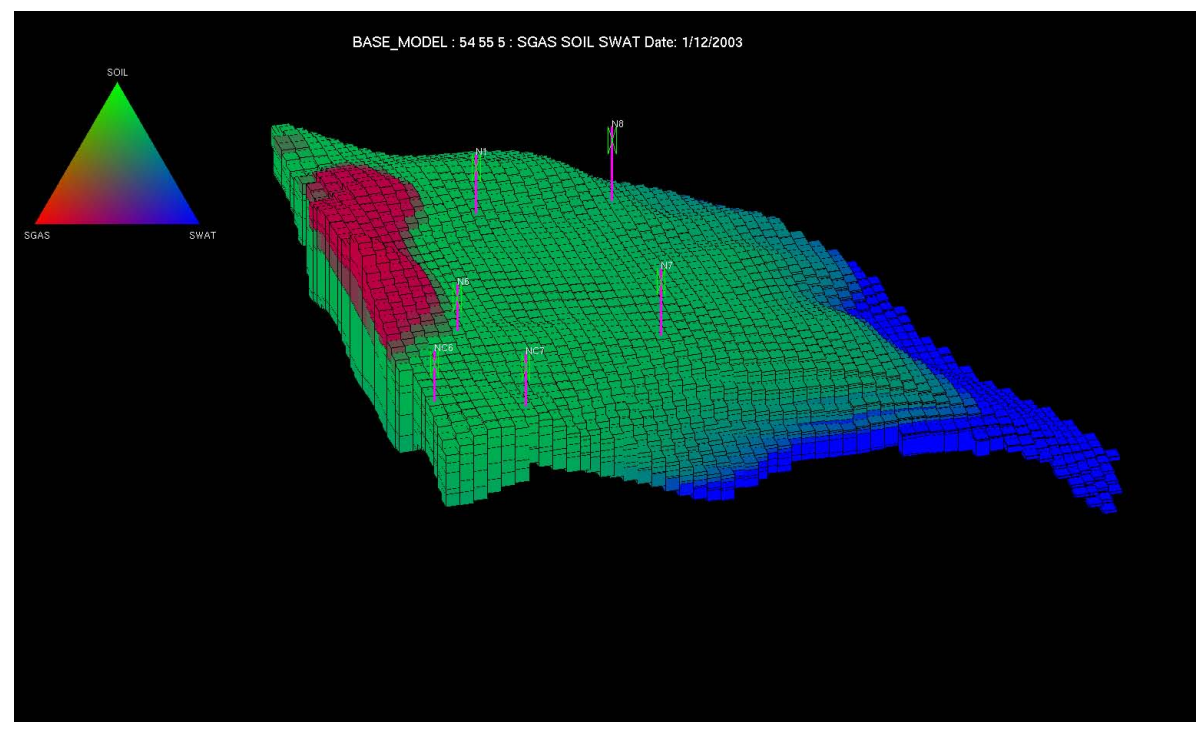

Figure 2: Three dimensional view of the realistic reservoir showing the oil saturation; the blue and red colours indicating high and low saturation respectively. 
The location and perforation intervals of one water injection well was optimised. Three-dimensional optimisation occurred over all five layers and the completion depth was taken as the bottom layer for two-dimensional trials. Optimal COP and NPV occurs when the injection well is placed in the gas cap to displace oil downwards to the production wells.

\subsection{Performance Measures}

Clerc (2011) proposes that a mean alone is insufficient to measure performance as a single high performing run is sufficient for a solution. Consequently, plots of average value found against unique function evaluations are used. One standard deviation error bars are included for only some cases to improve clarity. In addition to these plots, three performance measures are considered, based on those presented in Bangerth et al. (2006).

Effectiveness Effectiveness is a simple measure of performance and is the mean value between trials of the best solution found as a percent of the global optimum or,

$$
\bar{f}=\frac{1}{N} \sum_{i=1}^{N} \frac{f\left(\hat{p}_{i}\right)}{f(p *)},
$$

where $f(p)$ is the value of solution $p, p *$ is the globally optimum solution, $\hat{p}_{i}$ is the best solution found in trial $i$ and $N$ is the number of trials for each algorithm configuration.

Efficiency Efficiency indicates how quickly the algorithm reaches a level of performance using the number of unique evaluations required to find a solution of at least $98 \%$ the best solution value found, averaged between trials or

$$
\bar{L}=\frac{1}{N} \sum_{i=1}^{N} \frac{L_{i}^{98}}{M},
$$

where, additionally, $L_{i}^{98}$ is the number of unique function evaluations required to find solution $q$ such that $f(q) \leq 0.98 f\left(\hat{p}_{i}\right)$ for trial $i$ (for minimisation) and $M$ is the total number of function evaluations per trial.

Reliability Reliability indicates the expected minimum performance for a given number of optimisation runs by taking the effectiveness of a certain percentile of trials or,

$$
\varphi^{k}=\frac{f\left(\hat{p}_{k \%}\right)}{f(p *)},
$$

where, $\hat{p}_{k \%}$ is the lowest value required to reach the top $\mathrm{k}^{\text {th }}$ percentile of trials when ranked by their best solution.

All objective functions have been normalised to range from zero to one and only unique function evaluations are counted.

\section{Algorithm Configuration}

Four algorithms were implemented for testing, including assorted hill climbing methods, simulated annealing, genetic algorithm and particle swarm optimisation. A random search was also implemented and is included in results for comparison. Each implementation is briefly described here.

\subsection{Hill Climbing Algorithm}

This algorithm was implemented in Matlab (MATLAB, 2010) using three search patterns and three test-distance/stepsize methods based on developments in Spall (1992). The search patterns include searching all neighbouring points; searching at random, then moving as soon as a better solution is found; and searching based on the direction of the last step: opposite directions then orthogonal directions. The three stepping methods are presented in Table 2 where $c_{k}$ and $a_{k}$ are test distance and nominal step-size, initialised to half the minimum domain width; alpha $=0.602$ and gamma $=0.101$ are constants to control how quickly the search distance contracts; $k$ is an iteration counter; and $\frac{\Delta f}{c_{k}}$ is a linear gradient estimate of the current location using the test location. Further details of the SPSA test-distance/step-size can be found in Bangerth et al. (2006).

A penalty method, setting an infeasible solution's objective function to Matlab's 'inf' value is used to prevent the search leaving the feasible region. A random restart was implemented once the algorithm reached local optimality. 
Table 2: Configurations Tested for the Hill Climbing Algorithm

\begin{tabular}{c|ccc} 
& Search Method & Test Distance & Step Size \\
\hline Standard & All neighbouring & 1 & 1 \\
Random & Random neighbours & 1 & 1 \\
Orthogonal Search & Orthogonal Search Pattern & 1 & 1 \\
SPSA (one way check) & Orthogonal Search Pattern & $c_{k}=\left\lceil\frac{c_{k-1}}{k^{\gamma}}\right\rceil$ & $a_{k} \frac{\Delta f}{c_{k}} ; a_{k}=\left\lceil\frac{a_{k-1}}{k^{\alpha}}\right\rceil$ \\
SPSA (Equal step size0 & Orthogonal Search Pattern & $c_{k}=\left\lceil\frac{c_{k-1}}{k^{\gamma}}\right\rceil$ & $c_{k}$
\end{tabular}

\subsection{Simulated Annealing}

The simulated annealing package used is 'Simulated Annealing Tools', provided by Richard Frost through Frost Concepts as a supplement to Salamon et al. (2002). It has been modified to provide sufficient output for testing and to offer a function handle consistent with the other algorithms used.

The 'Uniform' and 'Gaussian' probability perturbation functions and the 'fast annealing' schedule function from (Ingber, 1996) were implemented and the 'Geman' schedule function is supplied with the Simulated Annealing Tools. The supplied default 'metropolis' acceptance function was also used (Salamon et al., 2002). Each combination of these two probability perturbation functions and two schedule functions were tested.

The initial temperature, a variable determining how long the algorithm runs and how much the solution can move, is also varied to values of 2,3 , and 4 . A restriction method is used such that the algorithm will correct all generated solutions back into the feasible region. It is restarted with a new random point once cooling has passed a specified limit.

\subsection{Genetic Algorithm}

The genetic algorithm used for testing is the 'Binary and Real-Valued Simulation Evolution for Matlab' created by Houck et al. (1996). Like the simulated annealing, modifications were made to unify the function interface and produce the output required for testing. The same restriction method as the simulated annealing genetic algorithm is used. Beyond this it is used in its original form.

The genetic algorithm was tested with 'Decimal' and 'Binary' encoding and populations of 4, 8, 16, 25 and 50. The default options in the 'Binary and Decimal-Valued Simulation Evolution for Matlab' are used for the crossover and mutation functions for each encoding method. Specifically, 'Binary Encoding' uses 'Simple Crossover' combined with 'Binary Mutation' and 'Decimal Encoding' uses 'Arithmetic Crossover', 'Heuristic Crossover' and 'Simple Crossover' with 'Boundary Mutation' and 'Multi-Non-Uniform Mutation'.

\subsection{Particle Swarm Optimisation}

A PSO algorithm was developed in Matlab for this study. It was developed with a number of modifications from that presented in the seminal paper Kennedy and Eberhart (1995). Maximum velocity was replaced with inertial weight (Shi and Eberhart, 1998).

Two sets of boundary conditions were tested. These were the restriction method, as implemented in both previous algorithms, and a penalty method such that any infeasible solution was never attractive for other particles. This was named the 'let them fly' by Bratton and Kennedy (2007). Neighbourhoods were varied based on results from Kennedy (2002) and Kennedy and Mendes (2006). These are named in Table 3 with implementations described in the above papers.

Many properties exist for particle swarm optimisation with minimal benchmarking for reservoir applications. The standard configuration, described in Bratton and Kennedy (2007), is used unless otherwise stated. This has global and local attractions of $\phi_{g}=\phi_{p}=1.496172$ and an inertia of $\omega=0.72984$.

The population size used was smaller due to a small search space and the use of unique function evaluations limiting iterations.

Stability was then assessed: tests were performed with 'let them fly' boundaries, Neumann neighbourhood and 3 particles per dimension and $\phi_{p}, \phi_{g}$ and $\omega$ were varied independently. $\omega$ was varied to $0.5,0.9$ and 1.1 ; $\phi_{p}$ and $\phi_{g}$ to 1.0, 2.0, 5.0. Finally, with 'set to nearest' boundaries, 'Neumann' neighbourhood, 2 particles per dimension and $\omega=0.72984$ both $\phi_{p}$ and $\phi_{g}$ were together varied to 1.0, 2.0 and 5.0.

\subsection{Comparisons}

The best algorithms of each category are compared. These include a genetic algorithm with binary encoding and a population of 4; a simulated annealing variation with 'Uniform' perturbation, 'Geman' schedule and initial temperature of 3; particle swarm optimisation schemes with the 'gbest' and 'lbest' neighbourhoods and 'let them 
Table 3: Configurations Tested for PSO

\begin{tabular}{c|ccc} 
Name & Boundary Method & Neighbourhood & Particles Per Dimension \\
\hline Standard & Set to nearest & Neumann & 2 \\
lbest neighbourhood & Set to nearest & lbest & 2 \\
gbest neighbourhood & Set to nearest & gbest & 2 \\
euclidean & Set to nearest & Euclidean & 2 \\
Let The Fly Boundaries & Let Them Fly & Neumann & 2 \\
ltf lbest 2ppd & Let Them Fly & lbest & 2 \\
ltf lbest 3ppd & Let Them Fly & lbest & 3 \\
ltf lbest 3ppd & Let Them Fly & lbest & 4
\end{tabular}

fly' boundary conditions; and the SPSA variation of the hill climbing algorithm. For initial seeding, a binary encoded genetic algorithm with a population of 25 was also included due to it's considerably high performance.

\section{Results}

Each of the algorithms were examined independently. The best configurations of each were then compared before seeded initialisation is introduced.

\subsection{Hill Climbing}

Variation in the realistic reservoir cases were considerably more than the synthetic cases, as shown in Figure 3. The standard performs worst in all three metrics. Considering performance here is measured against unique function evaluations it is clear that the additional evaluations of all the neighbouring points disadvantaged this method. The SPSA algorithms have very comparable performance to the orthogonal search suggesting the effect of step size is minimal for this application.

Hence, the performance seems to be most heavily influenced by the search pattern; often half a standard deviation, sometimes exceeding one, separates these. This trend is consistent over all cases.
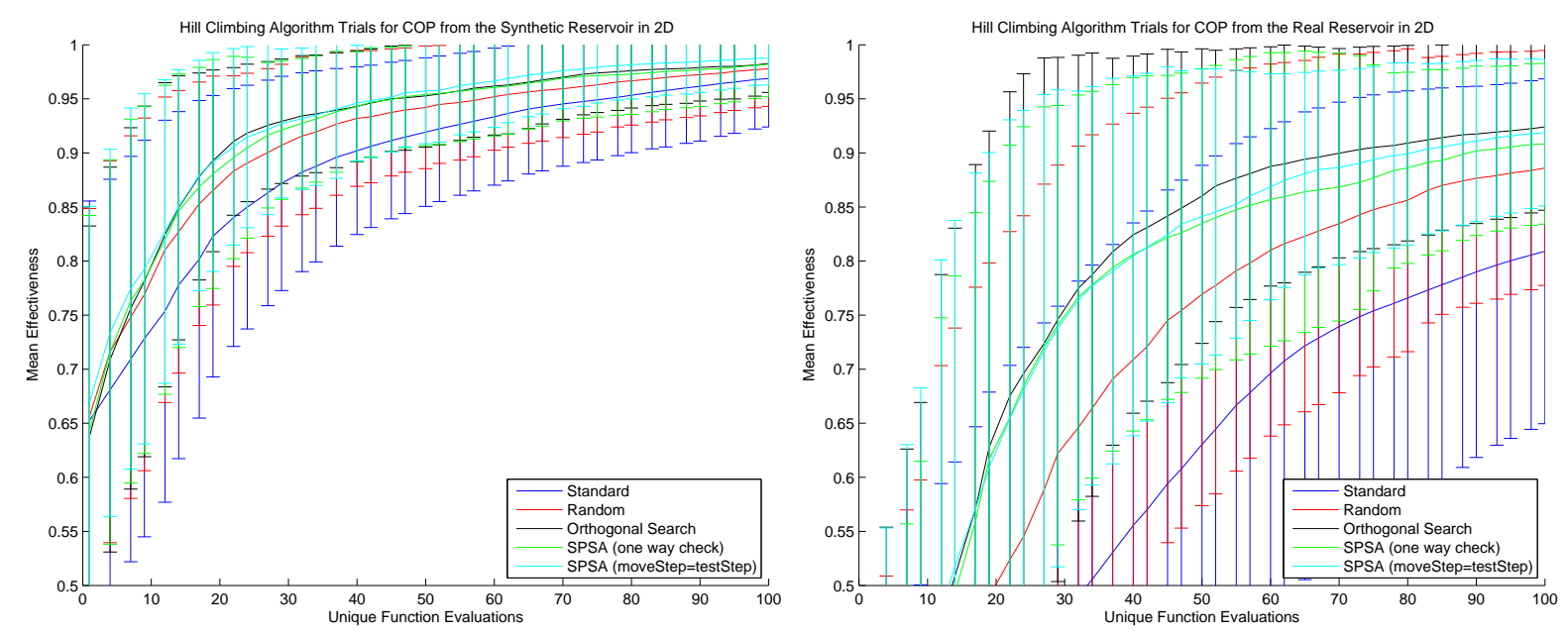

Figure 3: Performance of hill climbing algorithms for cumulative oil production of the synthetic (left) and realistic (right) reservoirs in two dimensions.

\subsection{Simulated Annealing}

There is large variation in algorithm performance for the realistic and three dimensional reservoir cases, exceeding one standard deviation at times, but much less in the synthetic and two dimensional cases, shown in Figure 4.

It appears that in most cases, for any pairing, the 'Uniform' and 'Geman' perform better than 'Normal' and 'Fast' respectively, regardless of initial temperature. Strangely, however, this trend is not only less defined for the synthetic reservoir case but largely reversed for the two dimensional cases, also shown in Figure 4. This is likely a 
consequence of the modality of each problem and suggests that the larger perturbations and more frequent restart of 'Normal' and 'Fast' annealing are better able to overcome local optima.
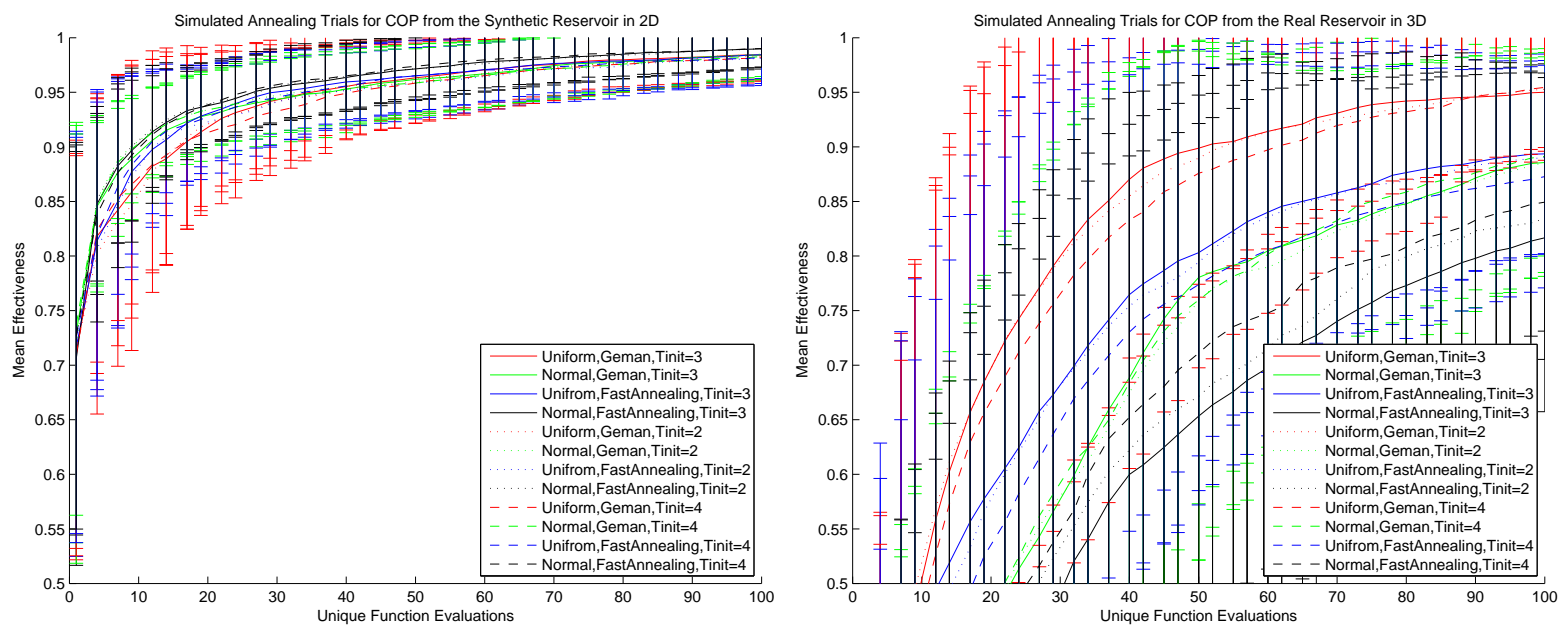

Figure 4: Performance of simulated annealing algorithms for cumulative oil production of the synthetic (left) and realistic (right) reservoirs in three dimensions.

\subsection{Genetic Algorithm}

The difference in performance between configurations is within one standard deviation in all but two cases.

Within these limits, there is a consistent trend in which the larger populations generally produce poorer performance and the binary encoding also appears less effective. One clear example is the cumulative oil production for the three dimensional realistic reservoir case; these results are presented in Figure 5 where the two trends are illustrated in the left and right plots respectively.

Overlaying these results the population size is more influential of the performance the encoding used.

The smaller populations can, however, perform worse in early time. As the genetic algorithm is initialised with random solutions, this implies that the smaller populations perform worse than a random search despite utilising information about the objective function after each iteration.
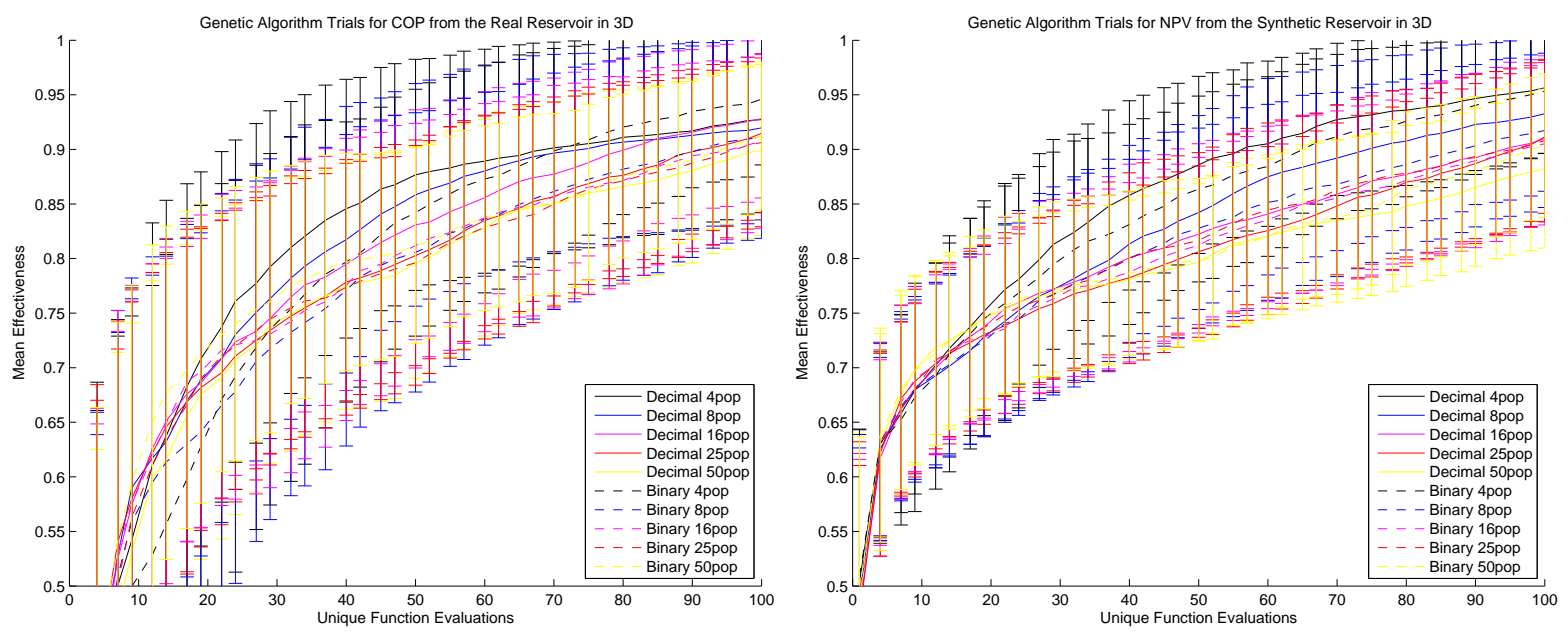

Figure 5: Performance of genetic algorithms for cumulative oil production of the realistic case (left) and NPC of the synthetic case (right) in three dimensions.

\subsection{Particle Swarm Optimisation}

Performance varies minimally, in the order of one tenth of a standard deviation and never exceeding half, within a reasonable parameter range: for the resolution of this study, 1.0 to 2.0 for personal and global best attractions 
and 0.5 to 0.8 for inertia weights. In Figure 6 both plots show this. Such stability improves the confidence of applying this method in industry.

Like genetic algorithms, smaller population sizes tend to perform better in late time, although there is no significant difference in early time. This behaviour is consistent between all trials and suggests, for these cases, a population smaller than recommended in literature is more appropriate.

'Let them fly' boundaries outperformed the restriction boundaries on all two dimensional cases but under performed in three dimensions as shown in Figure 7. This may be attributed to particles frequently leaving the small feasible region in the third dimension: five and fourteen layers deep. Under these conditions the centre favouring restriction boundaries perform better.

The 'gbest' neighbourhood performs well in short time but begins to plateau at later time. Table 4 supports this conclusion as the efficiency score of around 50 shows that even after 600 more evaluations a better solution is not found. The less connected 'lbest' neighbourhood finds a better solution after 70 evaluations and will ultimately find a solution $6 \%$ better $95 \%$ of the time.
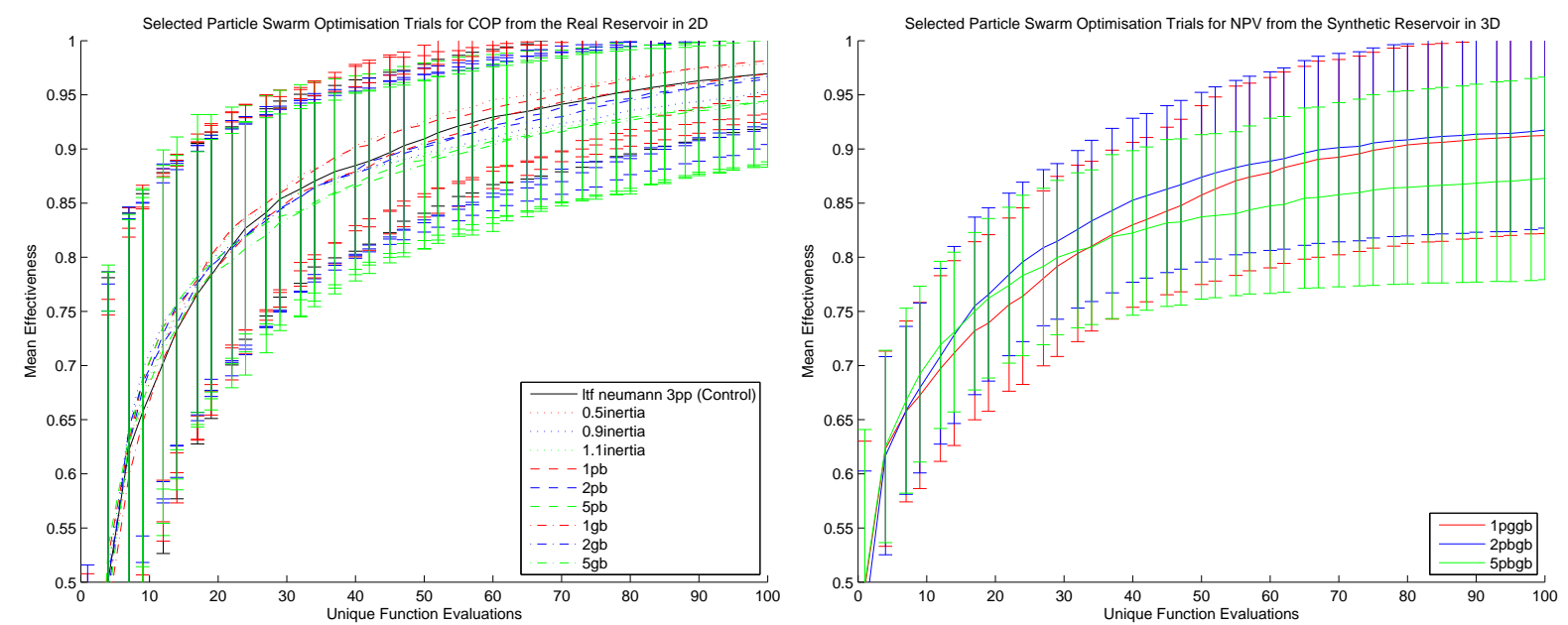

Figure 6: Performance of PSO algorithms with varying parameters for the cumulative oil production of the realistic case in two dimensions (left) and the NPC of the synthetic case in three dimensions (right).
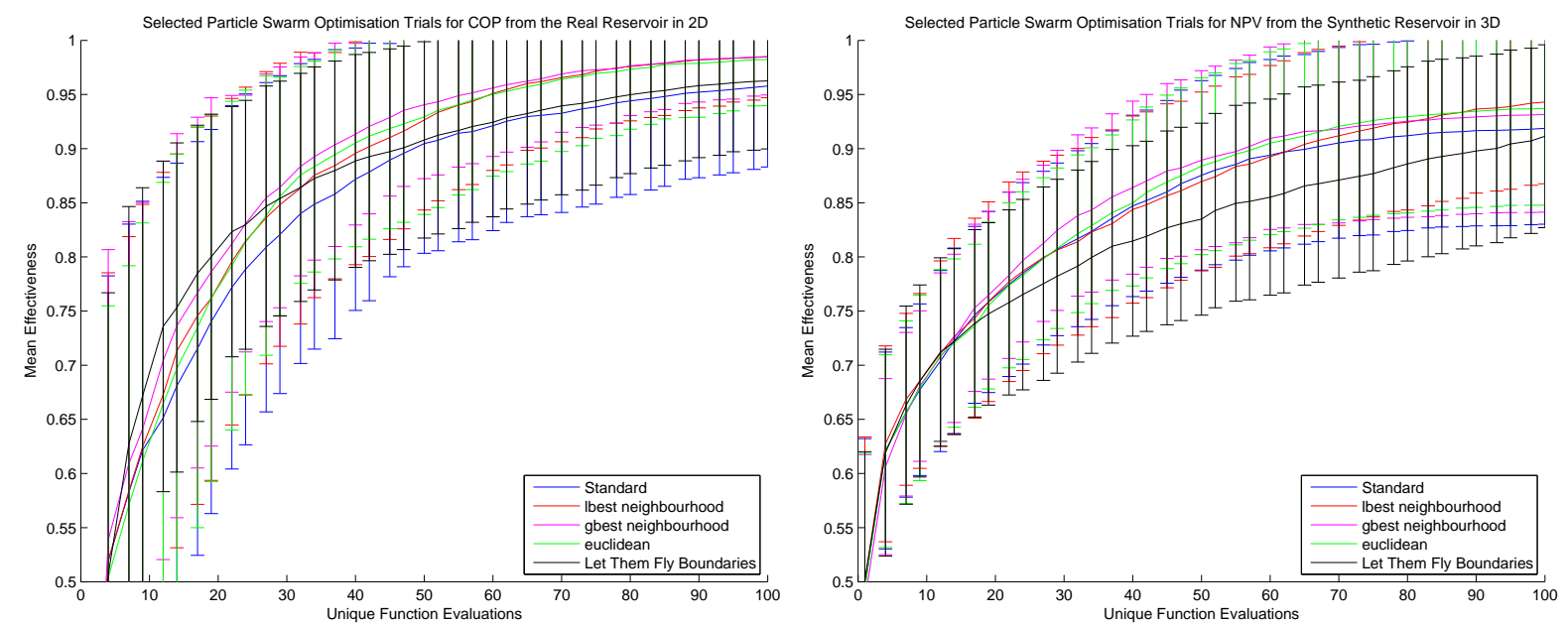

Figure 7: Performance of PSO algorithm with varying boundary methods and neighbourhood topologies for the cumulative oil production of the realistic case in two dimensions (left) and NPV of the synthetic case in three dimensions (right).

\subsection{Comparison}

This section compares the consistently best configurations of each algorithm, specified in Section 3. A random search is included for reference. 
Table 4: Performance Comparison of PSO Neighbourhoods for NPV in the 3-D Synthetic Reservoir Case

\begin{tabular}{l|r|cccc} 
Algorithm & Unique Evaluations & Effectiveness & Efficiency & Reliability $50 \%$ & Reliability 95\% \\
\hline 'lbest' neighbourhood & 75 & 0.94 & 52.73 & 0.97 & 0.82 \\
& 150 & 0.97 & 70.27 & 1.00 & 0.88 \\
& 750 & 0.97 & 71.08 & 1.00 & 0.88 \\
\hline \multirow{2}{*}{ 'gbest' neighbourhood } & 75 & 0.95 & 44.20 & 0.99 & 0.82 \\
& 150 & 0.96 & 51.37 & 1.00 & 0.82 \\
& 750 & 0.96 & 51.55 & 1.00 & 0.82
\end{tabular}

The let them fly, 'lbest' PSO is competitive in most instances and the top performer in some. Both the decimal encoding genetic algorithm and other PSO variants perform well, sometimes best, but lack consistency as seen in Figures 8 and 9. The binary encoded genetic algorithm performs in the top two in every reservoir case but often deteriorates in time, suggesting premature convergence.

Although simulated annealing improves with more function evaluations it is rarely the best algorithm at any stage. It requires a minimum of 20 function evaluations and as many as 50 to begin outperforming a random search. Table 5 shows this trend; after performing worse in all metrics at 50 evaluations it recovers after 100 evaluations where it is tied second equal for effectiveness but also has the best $50 \%$ reliability and third best $95 \%$ reliability. The efficiency shows that it more consistently finds the optimum after about 85 iterations where as the genetic algorithm and particle swarm optimisation converge prematurely after 50 and 32 evaluations respectively.

Table 5: Performance of Selected Algorithms for COP in the 2-D Synthetic Reservoir Case

\begin{tabular}{|c|c|c|c|c|c|}
\hline Algorithm & Unique Evaluations & Effectiveness & Efficiency & Reliability $50 \%$ & Reliability $95 \%$ \\
\hline \multirow[t]{3}{*}{ GA - Binary 4pop } & 50 & 0.96 & 24.86 & 0.95 & 0.90 \\
\hline & 100 & 0.98 & 46.34 & 0.98 & 0.94 \\
\hline & 500 & 0.98 & 50.03 & 1.00 & 0.94 \\
\hline \multirow{3}{*}{$\begin{array}{l}\text { SA - Uniform,Geman, } \\
\mathrm{T}_{\mathrm{i}}=3\end{array}$} & 50 & 0.94 & 25.16 & 0.95 & 0.85 \\
\hline & 100 & 0.98 & 47.52 & 1.00 & 0.89 \\
\hline & 500 & 1.00 & 84.67 & 1.00 & 1.00 \\
\hline \multirow[t]{3}{*}{ PSO - ltf lbest 2ppd } & 50 & 0.96 & 26.30 & 0.98 & 0.88 \\
\hline & 100 & 0.97 & 32.30 & 1.00 & 0.88 \\
\hline & 500 & 0.98 & 32.47 & 1.00 & 0.88 \\
\hline \multirow{3}{*}{$\begin{array}{l}\text { Hill Climb - SPSA } \\
\text { (move=test) }\end{array}$} & 50 & 0.96 & 24.57 & 0.95 & 0.88 \\
\hline & 100 & 0.99 & 42.98 & 1.00 & 0.95 \\
\hline & 500 & 1.00 & 66.33 & 1.00 & 1.00 \\
\hline
\end{tabular}

The hill climbing algorithm performs similarly to simulated annealing although often with worse performance in early time. In two dimensions it is competitive in late time, as seen in Figure 8, however, in three dimensions it often finds the optimum well after other algorithms. The performance is still trailing in Figure 9 but the 750 evaluations line in Table 6 shows both hill climbing and the genetic algorithm found the optimal solution but in 254 and 89 evaluations respectively.

\subsection{Initial Seeding}

The hill climbing algorithm's performance increased for all cases, in agreement with (Zandvliet et al., 2008). There was a noticeable delay before considerable improvement in the three dimensional synthetic case, as shown in Figure 10. This is a consequence of the approximations providing little information until the cell depth, which captures the most significant trend. This is verified by inspection of Appendix B.

The simulated annealing results also improved to become competitive with the PSO and often the best performing algorithm in the realistic reservoir cases. This suggests that this algorithm also effectively improves on, if not requires, existing solutions. Both these improvements are understandable as single point methods tend to explore a particular region exclusively so can be easily guided to the best region early.

PSO exhibits an unexpected behaviour in three of the synthetic reservoir cases: a sharp improvement exceeding 90\% within the first 10 evaluations, and then a large plateau in improvement. This can be shown in Figure 11. This may be explained by initialisation near a high value region, so that area is consistently found in short time. 
Table 6: Comparison of Algorithms for NPV in the 3-D Realistic Case

\begin{tabular}{l|r|cccc} 
Algorithm & Unique Evaluations & Effectiveness & Efficiency & Reliability $50 \%$ & Reliability $95 \%$ \\
\hline Random Search & 30 & 0.75 & 13.91 & 0.74 & 0.65 \\
& 75 & 0.81 & 36.91 & 0.80 & 0.72 \\
& 150 & 0.84 & 65.16 & 0.83 & 0.75 \\
& 750 & 0.93 & 335.46 & 0.93 & 0.85 \\
\hline Binary 4pop & 30 & 0.82 & 21.88 & 0.83 & 0.68 \\
& 75 & 0.93 & 47.06 & 0.98 & 0.84 \\
& 150 & 0.98 & 74.31 & 1.00 & 0.88 \\
Hill Climb - SPSA & 750 & 1.00 & 89.47 & 1.00 & 1.00 \\
(move=test) & 30 & 0.61 & 21.32 & 0.57 & 0.47 \\
& 75 & 0.80 & 54.04 & 0.82 & 0.55 \\
& 150 & 0.91 & 91.08 & 0.88 & 0.82
\end{tabular}
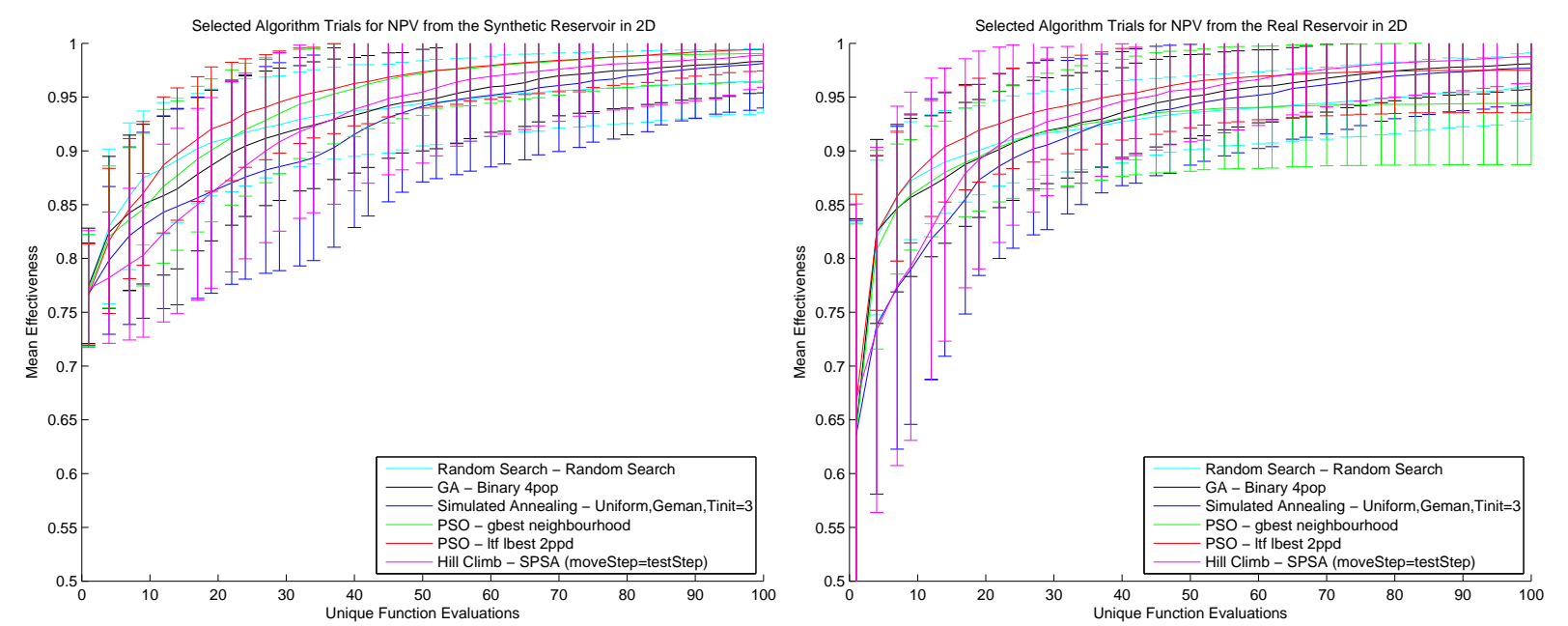

Figure 8: Performance of selected algorithms over the synthetic (left) and realistic (right) cases for NPV in two dimensions.

This behaviour was found to be independent of swarm size but is non-existent for the real cases, such as Figure 10, which makes it likely to be coincidental. After this time, improvement appears to be consistent with the previous behaviour and it is able to find the optimal solution consistently and at a time competitive with the simulated annealing as per the efficiency metric shown in Table 7.

The performance of the genetic algorithm is reduced considerably in the realistic reservoir cases. A larger population of 25 is also included as the best performing seeded genetic algorithm, however, the performance was still well below that of the other algorithms, observed in Figure 11, and the unseeded algorithm. This degradation is much less for the synthetic cases, shown in Figure 10.

\section{Discussion}

The genetic algorithm often does not converge to the optimal solution. A more volatile mutation may remedy this although it may also reduce performance. Particle swarm optimisation is competitive with the genetic algorithm and showed stable results for the test cases. Simulated annealing and the hill climbing algorithms were only competitive after many function evaluations and worse than a random search with few. This is in accordance with Bangerth et al. (2006): each local optimum is exploited, requiring around 30 evaluations, before reinitialisation; performance could be far better than any other algorithm if one of the first few solutions are within the optimal convex region, or very poor otherwise. In contrast, the population algorithms, that explore many points before exploitation, produce a more conservative search.

All algorithms except for the genetic algorithm improved with the use of optimal solution approximations to guide their initialisation. Simulated annealing became competitive with the PSO whereas the genetic algorithm 

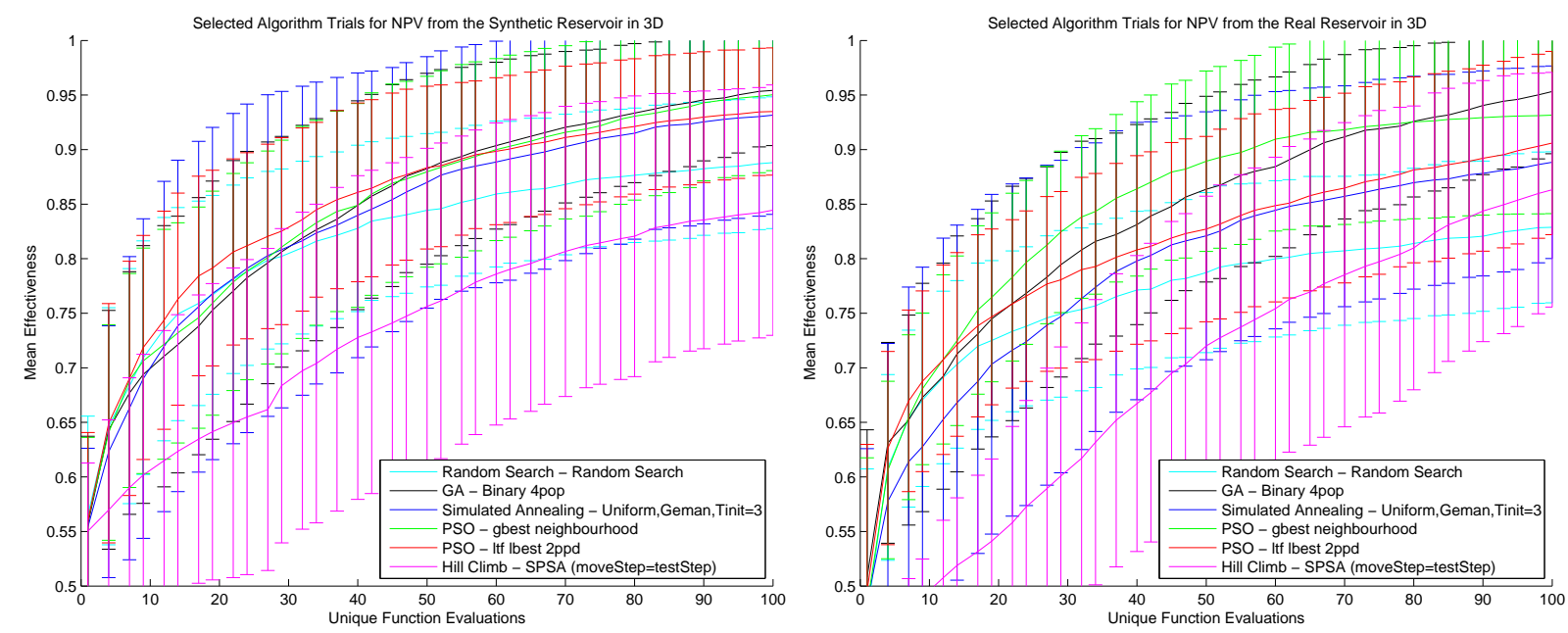

Figure 9: Performance of selected algorithms over the synthetic (left) and realistic (right) for NPV in three dimensions.
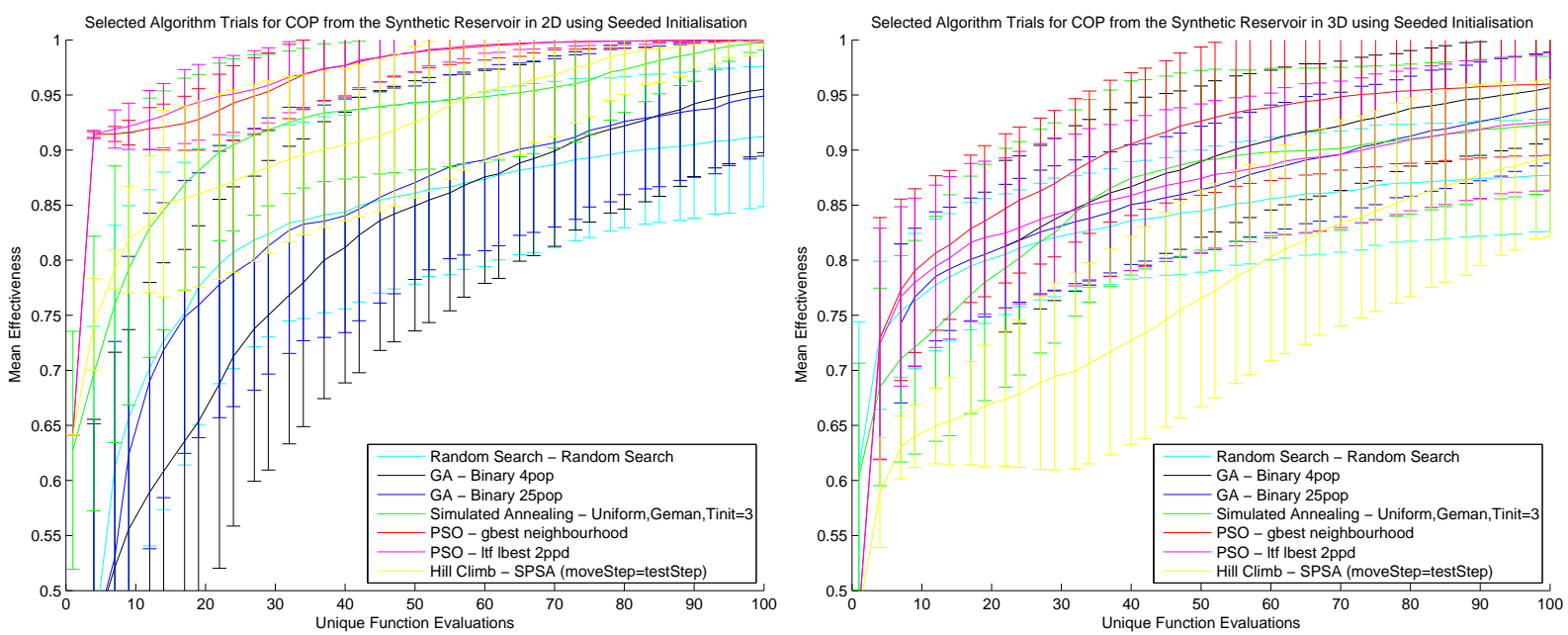

Figure 10: Performance of selected algorithms with initialisation seeding for the cumulative oil production of the synthetic case in two (left) and three (right) dimensions.

became relatively worse. This will be a consequence of how solutions interact within the algorithm. Both singlesolution algorithms are already designed to improve on a previous solution; PSO will gravitate all particles around the best known solution; where as genetic algorithms generate new solutions based on a population of existing solutions that is designed to efficiently cover the search space. Montes et al. (2001) explained this behaviour as the trade off between having high value points and genetic diversity: experimentally, genetic diversity is of greater importance.

It was found for these cases, the most effective population size is much less than that proposed in other literature. This is likely due to the small search space. It is reasonable to think that a larger reservoir may be more effectively searched with a larger population, however, with an excessive population, similar to the larger populations in these studies, the algorithm will approach a random search. This would suggest a population size exists for maximum efficiency and my be defined by a population density within the search space.

Subtle changes of other parameters made little difference to performance. More significant changes, such as the cooling function for simulated annealing or genetic algorithm encoding, often generated an advantage for limited cases. The overall performance varied little, however, even between algorithms: any mean was rarely more than one standard deviation from another.

Differences were often more pronounced after more function evaluations which may be evidence of a compounding effect of an algorithms behaviour. This would make the algorithm choice more significant in larger and more complex problems that require more function evaluations to sufficiently search.

Considering all these algorithms have had reported success in literature it is likely that each is effective in 

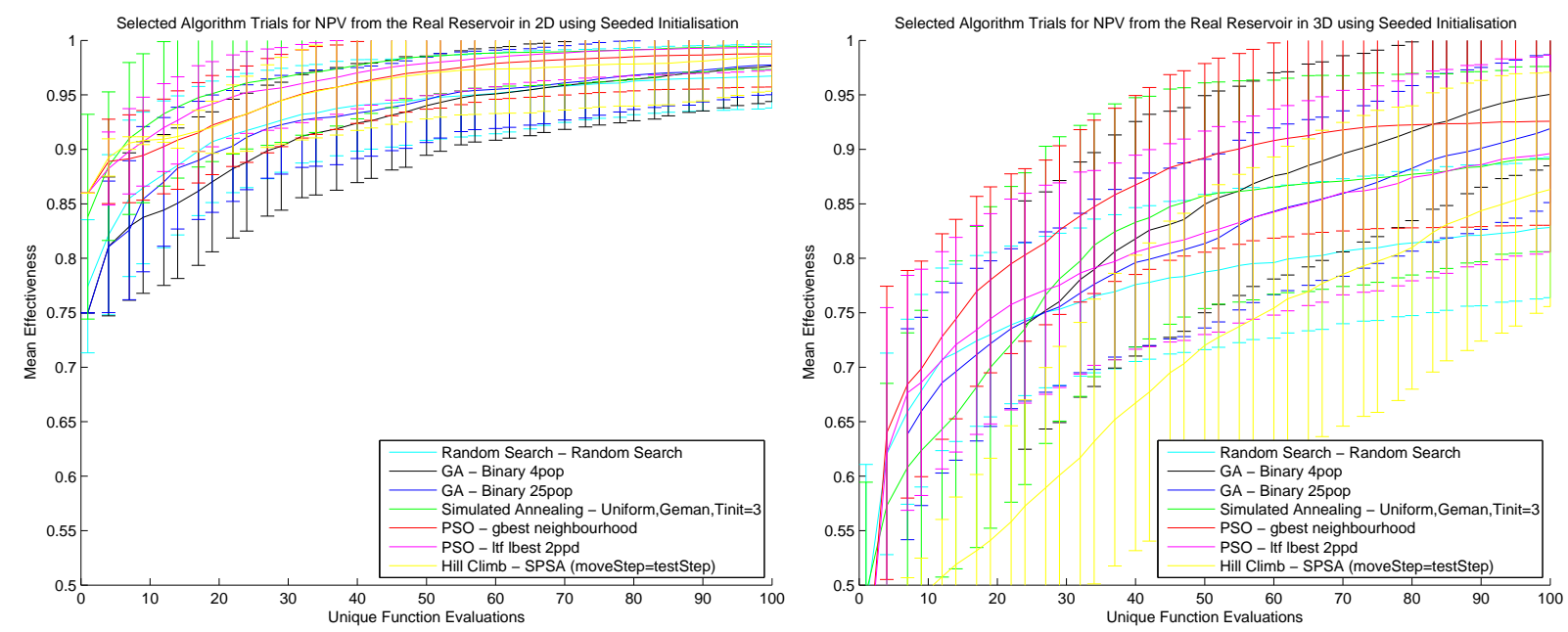

Figure 11: Performance of selected algorithms with initialisation seeding for the NPV of the real case in two (left) and three (right) dimensions.

Table 7: Performance of Algorithms with Initialisation Seeding for COP in the 2-D Realistic Reservoir Case

\begin{tabular}{l|r|cccc} 
Algorithm & Evaluations & Effectiveness & Efficiency & Reliability $50 \%$ & Reliability $95 \%$ \\
\hline GA - Binary 4pop & 500 & 0.98 & 65.92 & 1.00 & 0.91 \\
SA - Uniform,Geman,Ti=3 & 500 & 1.00 & 34.41 & 1.00 & 1.00 \\
PSO - ltf lbest 2ppd & 500 & 1.00 & 35.06 & 1.00 & 1.00 \\
Hill Climb - Orthogonal & 500 & 1.00 & 57.42 & 1.00 & 1.00 \\
Hill Climb - SPSAvar & 500 & 1.00 & 57.66 & 1.00 & 1.00
\end{tabular}

certain circumstances but results may not be transferable. Hence, extrapolation from these or other studies should be done cautiously and ideally verified experimentally. This would also suggest a method of characterising reservoirs and measuring performance against these characterisations be hugely beneficial in both selecting appropriate algorithms and understanding algorithm behaviour for the design of new algorithms.

\section{Conclusion}

It is clear from literature that some method of benchmarking is required to compare the many algorithm variations used for reservoir optimisation. This is attempted here by testing a selection of the most common algorithms against two, simple, single well-placement reservoir problems.

The genetic algorithm, as the most popular method in literature, and particle swarm optimisation both performed well in most cases tested. Both simulated annealing and the hill climbing algorithms perform poorly, often worse than a random search until after as many as 100 evaluations. All algorithms except for the genetic algorithm improved with the incorporation of a priori knowledge, particularly simulated annealing which became competitive with the PSO.

Based on these results, particle swarm optimisation is recommended as it is the most durable with and without guided initialisation. Simulated annealing could also be considered, although guided initialisation is almost essential otherwise performance is worse than a random search for a considerable number of evaluations. Seeding, however, has variable results with population-based searches and should not be used in conjunction with genetic algorithms.

The conclusions drawn from these experiments are limited due to computational resources restricting the reservoir sample size to two and the problem formulation to the location of only a single well. Computational restrictions are common and is likely the cause of the systemic lack of algorithm benchmarking for reservoir optimisation. Still, some valuable insight can be gained from these experiments that could guide further investigation.

This study should be extended to contain more test cases, preferably covering a range of complexities and typical variations in formation, properties and configuration; and a more comprehensive selection of algorithms including interpolation methods, adjoint methods and hybrid methods. This could benchmark algorithms to vet new developments to ensure a high quality of those implemented. It also has the potential to provide more insight into algorithm behaviour and guide the design of such algorithms based on their relative improvement rates at 
different phases.

\section{References}

Aanonsen, S., Eide, A., Holden, L., and Aasen, J. (1995). Optimizing reservoir performance under uncertainty with application to well location. In SPE Annual Technical Conference and Exhibition.

Badru, O. and Kabir, C. (2003). Well placement optimization in field development. In SPE Annual Technical Conference and Exhibition.

Bangerth, W., Klie, H., Wheeler, M., Stoffa, P., and Sen, M. (2006). On optimization algorithms for the reservoir oil well placement problem. Springer Science.

Beckner, B. and Song, X. (1995). Field development planning using simulated annealing-optimal economic well scheduling and placement. In SPE Annual Technical Conference and Exhibition.

Bittencourt, A. and Horne, R. (1997). Reservoir development and design optimization. Paper SPE, 38895:5-8.

Bratton, D. and Kennedy, J. (2007). Defining a standard for particle swarm optimization. In Swarm Intelligence Symposium, 200\%. SIS 200\%. IEEE, pages 120-127. IEEE.

Ciaurri, D., Mukerji, T., and Durlofsky, L. (2011). Derivative-free optimization for oil field operations. In Yang, X.-S. and Koziel, S., editors, Computational Optimization and Applications in Engineering and Industry, volume 359 of Studies in Computational Intelligence, pages 19-55. Springer Berlin / Heidelberg. 10.1007/978-3-64220986-4_2.

Clerc, M. (2011). From Theory to Practice in Particle Swarm Optimization in the Handbook of Swarm Intelligence, pages 3-36. Springer.

Ding, D. (2008). Optimization of well placement using evolutionary algorithms. In SPE Europec/EAGE Annual conference and exhibition.

ECLIPSE (2009). 2009.1. Schlumberger, Houston, Texas.

Guyaguler, B., Home, R., Rogers, L., and Rosenzweig, J. (2002). Optimization of well placement in a gulf of mexico waterflooding project. SPE Reservoir Evaluation \& Engineering, 5(3):229-236.

Houck, C., J.Joines, and M.Kay (1996). A genetic algorithm for function optimization: A MATLAB implementation. ACM Transactions on Mathmatical Software.

Ingber, L. (1996). Adaptive simulated annealing (asa): Lessons learned. In Control and Cybernetics. Citeseer.

Kennedy, J. (2002). Population structure and particle swarm performance. In In: Proceedings of the Congress on Evolutionary Computation (CEC 2002, pages 1671-1676. IEEE Press.

Kennedy, J. and Eberhart, R. C. (1995). Particle swarm optimization. In Proc. IEEE International Conference on Neural Networks (Perth, Australia), pages IV: 1942-1948, IEEE Service Center, Piscataway, NJ.

Kennedy, J. and Mendes, R. (2006). Neighborhood topologies in fully informed and best-of-neighborhood particle swarms. Systems, Man, and Cybernetics, Part C: Applications and Reviews, IEEE Transactions on, 36(4):515519 .

Lo, K., Starley, G., and Holden, C. (1995). Application of linear programming to reservoir development evaluations. SPE Reservoir Engineering, 10(1):52-58.

MATLAB (2010). version 7.10 (R2010a). The MathWorks Inc., Natick, Massachusetts.

Montes, G., Bartolome, P., and Udias, A. (2001). The use of genetic algorithms in well placement optimization. In SPE Latin American and Caribbean Petroleum Engineering Conference.

Morales, A., Nasrabadi, H., and Zhu, D. (2011). A new modified genetic algorithm for well placement optimization under geological uncertainties. In SPE EUROPEC/EAGE Annual Conference and Exhibition.

Nakajima, L. and Schiozer, D. (2003). Horizontal well placement optimization using quality map definition. In Canadian International Petroleum Conference. 
Onwunalu, J. and Durlofsky, L. (2009a). Development and application of a new well pattern optimization algorithm for optimizing large scale field development. In SPE Annual Technical Conference and Exhibition.

Onwunalu, J. E. and Durlofsky, L. J. (2009b). Application of a particle swarm optimization algorithm for determining optimum well location and type. Springer Science.

Pan, Y. and Horne, R. (1998). Improved methods for multivariate optimization of field development scheduling and well placement design. In SPE Annual Technical Conference and Exhibition.

Poli, R., Kennedy, J., and Blackwell, T. (2007). Particle swarm optimization an overview. Springer Science.

Salamon, P., Frost, R., and Sibani, P. (2002). Facts, Conjectures, and Improvements for Simulated Annealing. Society for Industrial and Applied Mathematics, Philadelphia, PA, USA.

Sarma, P. and Chen, W. H. (2008). Efficient well placement optimization with gradient-based algorithms and adjoint models. SPE International.

Shi, Y. and Eberhart, R. (1998). A modified particle swarm optimizer. In Proc. of the 1998 IEEE Congress on Evolutionary Computation, Anchorage, AK.

Spall, J. (1992). Multivariate stochastic approximation using a simultaneous perturbation gradient approximation. Automatic Control, IEEE Transactions on, 37(3):332-341.

Tupac, Y., Almeida, L., Almeida, L., and Vellasco, M. (2007). Evolutionary optimization of oil field development. In Digital Energy Conference and Exhibition, Houston, Texas, U.S.A.

Wang, C., Li, G., and Reynolds, A. (2007). Optimal well placement for production optimization. In Eastern Regional Meeting, Lexington, Kentucky USA.

Zandvliet, M., Handels, M., van Essen, G., Brouwer, R., and Jansen, J. (2008). Adjoint-based well-placement optimization under production constraints. SPE Journal, 13(4):392-399.

Zangl, G., Graf, T., and Al-Kinani, A. (2006). Proxy Modeling in Production Optimization. In SPE Europec/EAGE Annual Conference and Exhibition, Vienna, Austria. 


\section{A Simulated Functions}
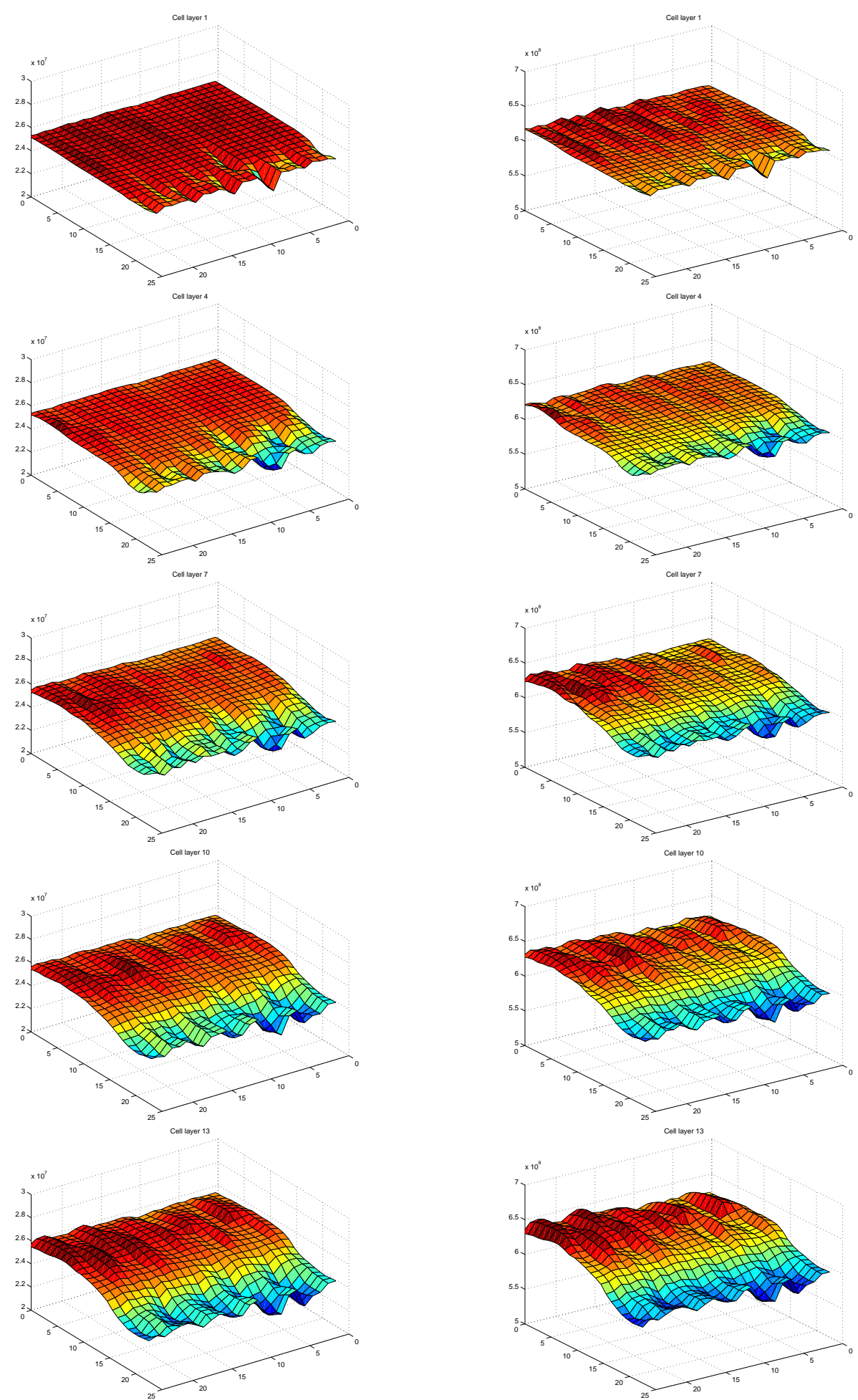

Figure 12: COP in barrels (left) and NPV in dollars (right) over the new well locations in the synthetic reservoir for five selected lower perforation limits. 

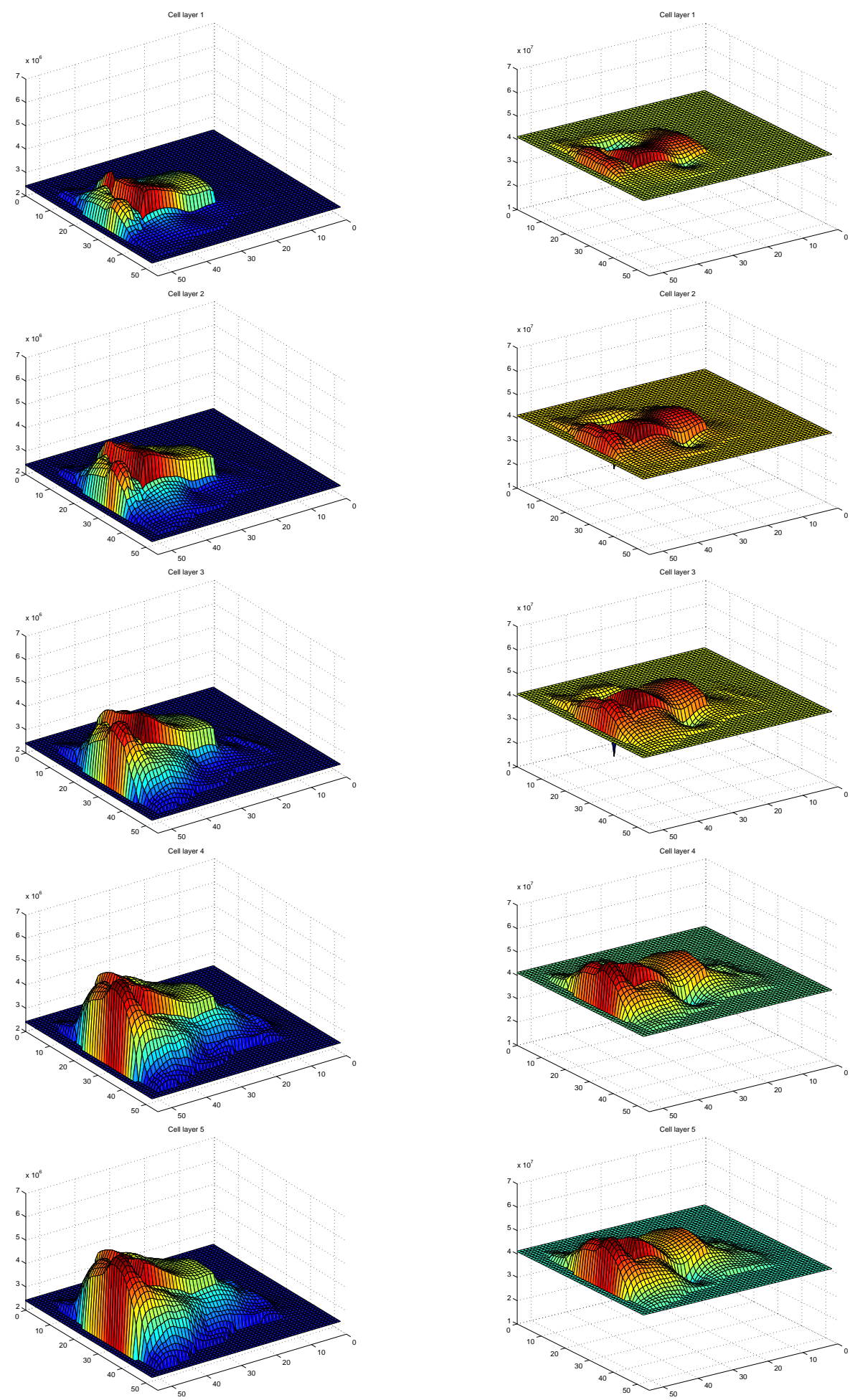

Figure 13: COP in barrels (left) and NPV in dollars (right) over the new well locations in the realistic reservoir for each lower perforation limit. 


\section{B Objective Function Approximations}
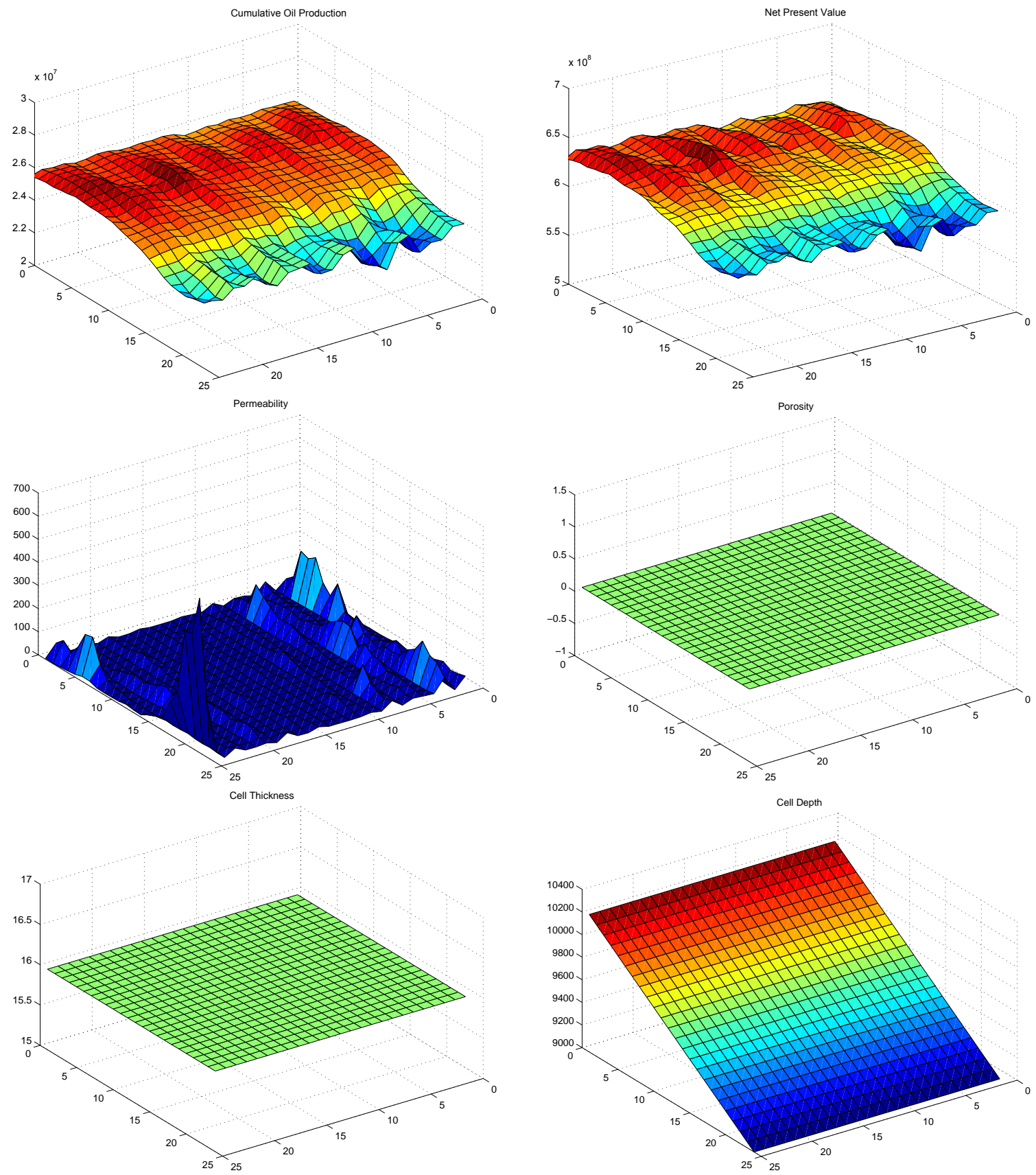

Figure 14: COP (top left) and NPV (top right) followed by the objective function approximations used for the fifth layer of the synthetic reservoir case. 

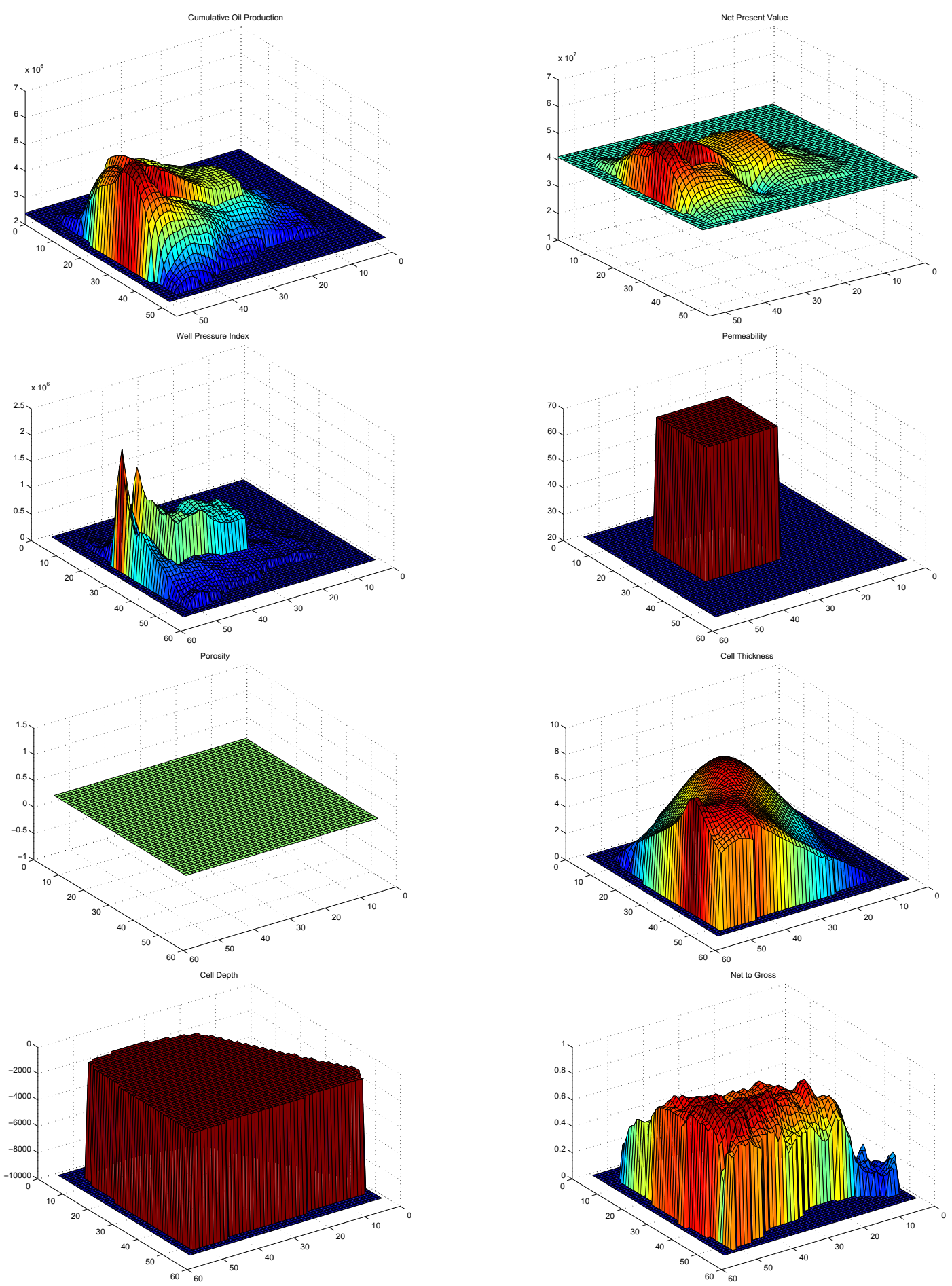

Figure 15: COP (top left) and NPV (top right) followed by the objective function approximations used for the fifth layer of the realistic reservoir case. 